\title{
MODULATION OF THE SHUBNIKOV-DE HAAS OSCILLATIONS BY THE EXCHANGE INTERACTION IN $\mathrm{Cd}_{3-x-y} \mathrm{Zn}_{x} \mathrm{Mn}_{y} \mathrm{As}_{2}$
}

\author{
W. LuBCZyŃski, J. Cisowski \\ Department of Solid State Physics, Polish Academy of Sciences, ul. Kawalca 3, 41-800 \\ Zabrze, Poland
}

AND J.C. PORTAL

CNRS-SNCI, Grenoble, France

(Received August 8, 1990)

\begin{abstract}
New measurements of the Shubnikov-de Haas ( $\mathrm{SdH}$ ) effect in semimagnetic semiconductor (SMSC) $\mathrm{Cd}_{3-x-y} \mathrm{Zn}_{x} \mathrm{Mn}_{y} \mathrm{As}_{2}$ with $x=0.09$ and $y=0.018$, using continuous magnetic fields up to $25 \mathrm{~T}$, are presented. The observed modulation of the amplitudes of the first and second SdH harmonics as a function of magnetic field is interpreted in terms of the three band Kane model with the exchange interaction included and taking into account the spin-dependent scattering which appears to be particularly important at high magnetic fields.
\end{abstract}

PACS numbers: $72.20 . \mathrm{My}$

The first study of the quantum transport in $\mathrm{Cd}_{3-x-y} \mathrm{Zn}_{x} \mathrm{Mn}_{y} \mathrm{As}_{2}$ has shown that the Landau spin subband energy states in this material are very strongly influenced by the exchange interaction between band electrons and the localized magnetic moment of $\mathrm{Mn}$ ions [1]. As a consequence of this interaction only one minimum of the first harmonic amplitude of the SdH oscillations has been found in the magnetic fields below $10 \mathrm{~T}$. In order to find other minima we have extended the range of the magnetic field up to $25 \mathrm{~T}$ by using the hybrid magnet in Grenoble [2].

For this study we have chosen the alloy with $x=0.09$ and $y=0.018$. The measurements have been performed in transverse configuration in the temperature range of 1.6-4.2 K. Independently, the magnetization, which practically saturates at about $15 \mathrm{~T}$, has been measured on the same material. The SdH traces, recorded 


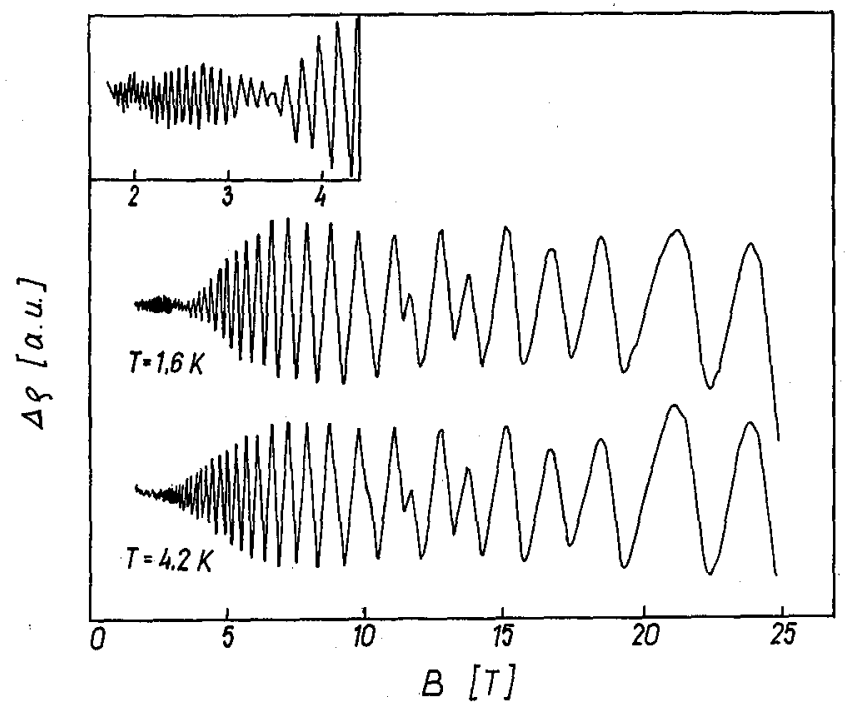

Fig. 1. SdH oscillations at two different temperatures for a sample of $\mathrm{Cd}_{2.892} \mathrm{Zn}_{0.09} \mathrm{Mn}_{0.018} \mathrm{As}_{2}$ with the electron concentration $4.05 \times 10^{24} \mathrm{~m}^{-3}$. The inset presents the low-field oscillations at $T=1.6 \mathrm{~K}$ in greater detail.

for a sample with the electron concentration $n=4.05 \times 10^{24} \mathrm{~m}^{-3}$ are presented in Fig. 1. As can be seen, there is only one node at about $3.5 \mathrm{~T}$ at $1.6 \mathrm{~K}$ (which disappears at higher temperature) whereas at higher magnetic fields a broad minimum is observed.

To analyse the complicated SdH pattern in the whole range of a magnetic field the least-squares fitting technique was used $[3,1]$. The first and second harmonic amplitudes obtained in such a way are presented in Fig. 2. It can be seen that in the investigated field range there are two minima of the first and also two minima of the second harmonic. Such modulation of the amplitudes is due to the factors $\cos (\pi \nu)$ and $\cos (2 \pi \nu)$ (where $\nu$ is the ratio between spin- and Landau splitting) for the first and second harmonic, respectively, following from the magnetic field dependence of the effective $g$-factor [4].

To describe our experimental data (circles in Fig. 2) we have applied the three band Kane model completed with the exchange interaction and we have taken into account the spin-dependent scattering already observed in $\mathrm{Cd}_{3-x-y} \mathrm{Zn}_{x} \mathrm{Mn}_{y} \mathrm{As}_{2}$ [1]. We have taken the value of the energy gap $E_{0}=-0.055 \mathrm{eV}$, whereas the other parameters are those of $\mathrm{Cd}_{3} \mathrm{As}_{2}$ [5].

The exchange interaction constants $\alpha$ and $\beta$ and $\delta T_{\mathrm{D}}=\left(T_{\mathrm{D}}^{+}-T_{\mathrm{D}}^{-}\right) / 2$ (where $T_{\mathrm{D}}^{+}$and $T_{\mathrm{D}}^{-}$are Dingle temperatures for spin-up and spin-down electrons, respectively) have been treated as fitting parameters. The best fitted values are: $\alpha=-0.4 \mathrm{eV}$ and $\beta=2.14 \mathrm{eV}$. what is consistent with the values found by us for other compositions. As far as $\delta T_{\mathrm{D}}$ is concerned, this quantity increases with the 
magnetic field (see Fig. 2) showing the importance of the spin-dependent scattering at high magnetic fields. For this reason, we have recently undertaken an attempt to describe this phenomenon [6].

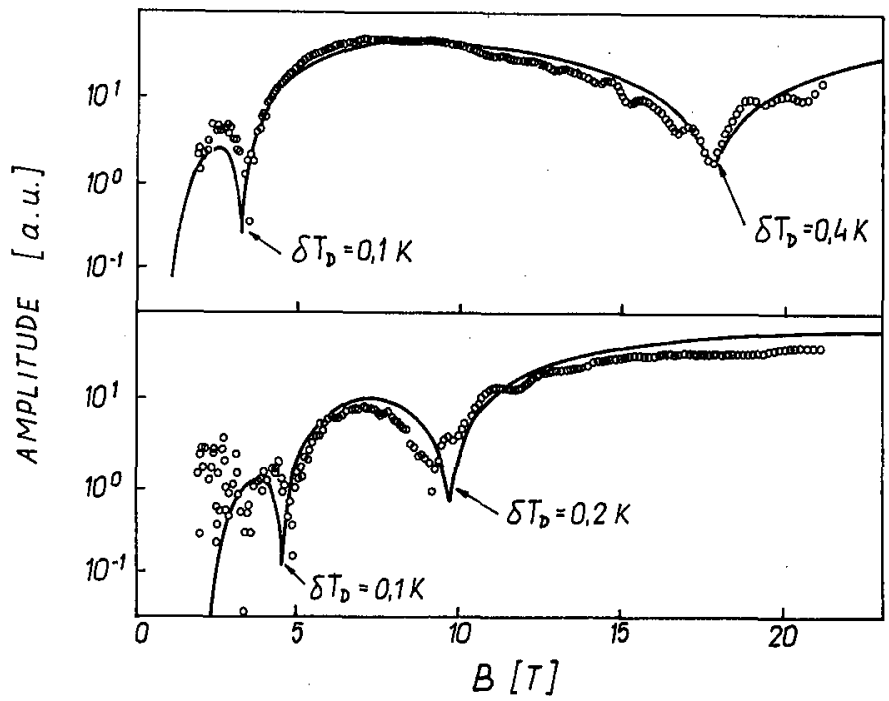

Fig. 2. The amplitude of the first (upper part) and second (lower part) harmonics of the SdH oscillations from Fig. 1 for $T=1.6 \mathrm{~K}$, obtained from the fitting procedure. The solid lines represent theoretical dependence (see the text).

\section{References}

[1] W. Lubczyński, J. Cisowski, J.C. Portal, W. Żdanowicz, Acta Phys. Pol. A73, 509 (1988).

[2] G. Landwehr, in Physics in High Magnetic Fields, Springer Series in Solid State Sciences, Vol. 24, eds. S. Chikazumi, N. Miura, Springer-Verlag, Berlin 1981, p. 2.

[3] M. Vaziri, R. Reifenberger, Phys. Rev. B 32, 3921 (1985).

[4] J. Kossut, in Semiconductors and Semimetals, Vol. 25, eds. J.K. Furdyna, J. Kossut, Academic Press, San Diego 1988, p. 183.

[5] F.A.P. Blom, in Narrow Gap Semiconductors Physics and Applications, in series Lecture Notes in Physics, Vol. 133, ed. W. Zawadzki, Springer-Verlag, Berlin, Heidelberg, New York 1980 p. 191.

[6] W. Lubczyński, J. Cisowski, J. Kossut, J.C. Portal, to be published. 